\title{
Continuous- and Dispersed-Phase Structure of Dense Nonevaporating Pressure-Atomized Sprays
}

\author{
G. A. Ruff, ${ }^{*}$ P.-K. Wu, $†$ L. P. Bernal,$\ddagger$ and G. M. Faeth $\S$ \\ University of Michigan, Ann Arbor, Michigan 48109
}

\begin{abstract}
The structure and breakup properties of the dense-spray region of nonevaporating pressure-atomized sprays were studied. The multiphase mixing layer near the injector exit was emphasized, considering large-scale (9.5$\mathrm{mm}$ injector diameter) water jets injected vertically downward in still room air. Phase-discriminating laser velocimetry and double-pulse holography were used to measure phase velocities and drop-size properties for both nonturbulent and turbulent jet exit conditions. Present test conditions involved two types of primary breakup: 1) aerodynamic breakup for nonturbulent jets where properties could be correlated using earlier aerodynamic breakup theories; and 2) turbulent breakup for turbulent jets where drop properties could be related to liquid turbulence properties. Both mechanisms yielded Weber numbers exceeding secondary drop breakup limits near the liquid surface. Significant effects of separated flow were observed for present test conditions; however, scaling analysis suggests reduced effects of separated flow at higher injector velocities and ambient pressures-largely due to finer atomization.
\end{abstract}

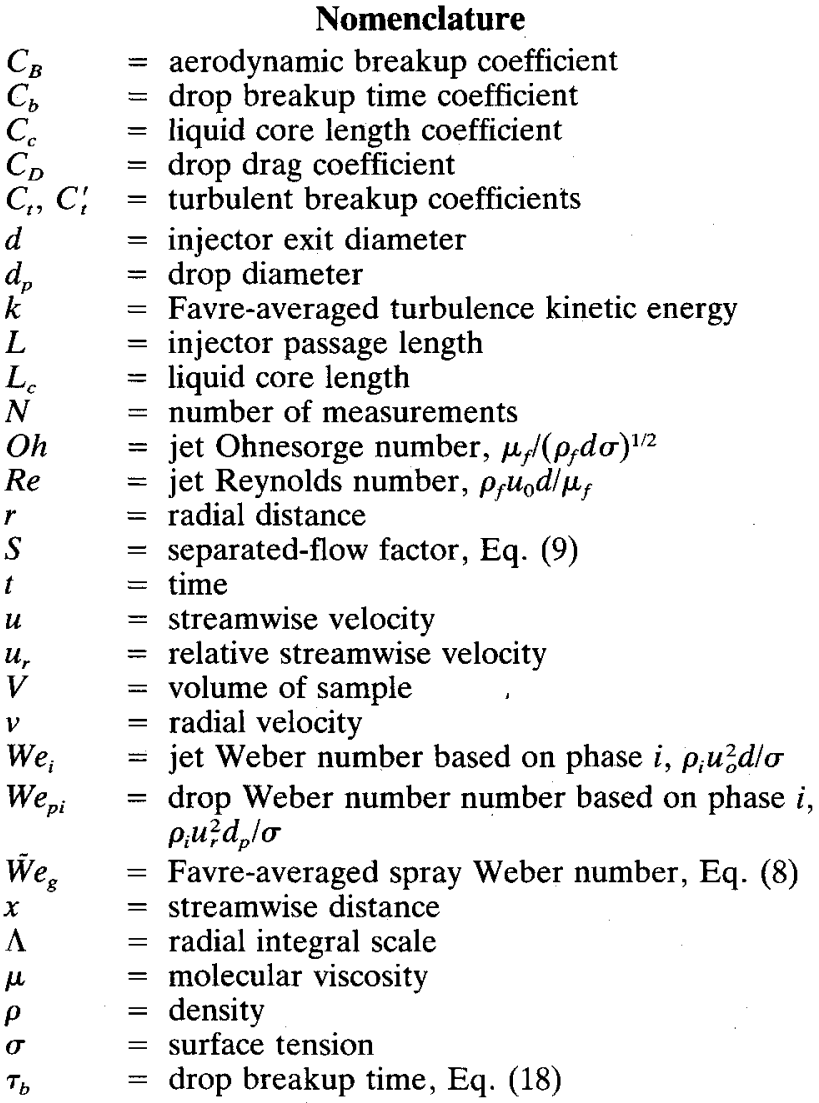

Presented as Paper 90-0464 at the AIAA 28th Aerospace Sciences Meeting, Reno, NV, Jan. 8-11, 1990; received April 20, 1990; revision received Feb. 20, 1991; accepted for publication Feb. 21, 1991. Copyright (C) 1991 by the American Institute of Aeronautics and Astronautics, Inc. All rights reserved.

*Graduate Assistant, Department of Aerospace Engineering; currently, Assistant Professor, Department of Mechanical Engineering, Drexel University, Philadelphia, PA 19104.

† Graduate Assistant, Department of Aerospace Engineering.

\#Associate Professor, Department of Aerospace Engineering. Member AIAA.

§Professor, Department of Aerospace Engineering. Fellow AIAA.

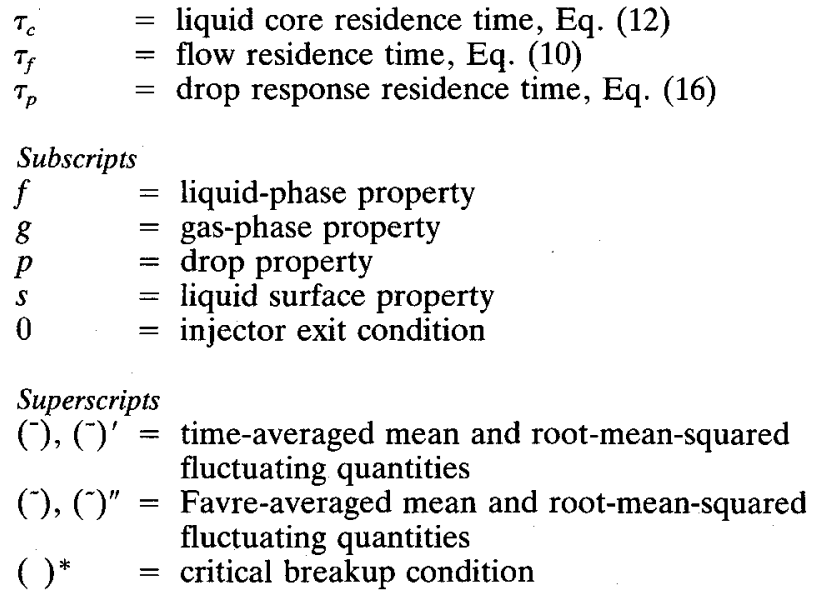

\section{Introduction}

HERE have been numerous efforts to develop methods to analyze spray processes. The present investigation seeks to contribute to this methodology by studying the dense-spray region near the exit of the injector passage for pressure atomization. Experiments were limited to relatively large-scale (9.5$\mathrm{mm}$ injector diameter) nonevaporating round water jets injected into still air at normal temperatures and pressures Earlier measurements of liquid volume fractions, entrainment rates, and dispersed-phase properties in the dense-spray region of these flows ${ }^{1,2}$ were extended to provide gas-phase and additional dispersed-phase properties. The new measurements, along with additional information obtained from the data of Ruff et al., ${ }^{1}$ were used to continue study of locally homogeneous-flow (LHF) analysis of the process, e.g., analysis based on the assumption of infinitely fast interphase transport rates so that both phases have the same velocity and are in thermodynamic equilibrium at each instant and point within the flow. Present measurements were limited to the atomization breakup regime where a multiphase mixing layer along the edge of the flow begins to develop right at the injector exit. $^{3.4}$ Jet exit conditions involved both slug and fully developed turbulent pipe flow because past work had shown sensitivity of dense-spray properties to liquid-phase turbulence levels. ${ }^{1,2}$

For atomization breakup, the flow near the injector exit involves a liquid core (much like the potential core of a single- 
phase jet), surrounded by a multiphase mixing layer that begins to develop right at the injector exit. ${ }^{3-5}$ The dense-spray region is normally considered to include both the liquid core and the multiphase mixing layer up to the point where the liquid core disappears. There have been several studies of the length of the liquid core, taken as the length of unbroken liquid extending from the passage exit. ${ }^{6-8}$ Findings indicate that this length is influenced by the breakup regime, turbulence properties at the injector exit, and the gas/liquid density ratio. For atomization breakup of typical liquids in gases at atmospheric pressure, however, the dense-spray region extends quite far from the injector, ca. 200-400 injector diameters. Thus, dense sprays are an important feature of spray injection processes due both to their extent and their influence on drop properties at the start of the dilute-spray region.

The multiphase mixing layer is also affected by the breakup regime, jet exit turbulence properties, and the density ratio of the flow. ${ }^{1,2,9-11}$ Ruff et al. ${ }^{1}$ provide information on liquidphase properties in the multiphase mixing layer for water jets in still air at atmospheric pressure, using double-pulse holography to find liquid element (drop) sizes and velocities. It was found that the multiphase mixing layer had liquid volume fractions less than $1 \%$ for their test conditions. The inner portion of the mixing layer contained large irregularly shaped liquid elements and drops while the proportion of spherical drops increased and drop sizes decreased with increasing radial distance, suggesting significant effects of secondary breakup in the flow. The velocities of large drops were generally much larger than small drops, implying that separated flow effects were important as well. Increased turbulence levels at the jet exit had a substantial effect on the structure of the flow, increasing the number and size of irregular liquid elements by promoting ejection of liquid from the liquid surface and increasing the width of the liquid-containing region of the multiphase mixing layer. Wu et al. ${ }^{9}$ also observed significant effects of jet exit conditions on the rate of spread of the outer edge of the multiphase mixing layer.

The complexities of dense sprays-involving stripping of liquid from the all-liquid core, the presence of irregular liquid elements, secondary breakup, and turbulent dispersion of drops toward the edge of the flow-make the LHF approximation attractive as a means of circumventing detailed descriptions of these phenomena. Ruff et al. ${ }^{2}$ found that LHF predictions were reasonably effective for estimating distributions of liquid volume fractions for atomization breakup in the region where mean liquid volume fractions were greater than 0.2 . However, the multiphase mixing layer dominates flow properties at lower liquid volume fractions and exhibits significant effects of separated flow, which limits the effectiveness of the LHF approximation. ${ }^{1}$ Other evaluations of the LHF approximation find varying degrees of success, at times yielding encouraging results and at other times overestimating the rate of development of the flow as the spray becomes dilute. ${ }^{5,9-12}$ Unfortunately, methods for determining conditions when the LHF approximation is appropriate for dense sprays have not been developed. The main difficulty is that success of the LHF approximation depends on drops being small enough to respond quickly to changes in gas properties, while information on both drop sizes and gas properties in dense sprays is very limited.

The objective of the present investigation was to seek a better understanding of the properties of dense sprays by studying both gas- and dispersed-phase velocities in the multiphase mixing layer for the same test conditions as Ruff et al. ${ }^{1,2}$ Coupled with the existing measurements of liquid-phase properties (drop sizes and velocities), this provides a relatively complete picture of the structure of these flows. Furthermore, knowledge of both drop size distributions and phase velocities allows direct evaluation of primary breakup properties, the propensity for secondary drop breakup, and quantitative estimates of effects of separated flow. Mean and fluctuating gas velocities near the edge of the multiphase mixing layer were measured using phase-discriminating laser velocimetry (LV), whereas double-pulse holography was used near the liquid surface where LV was no longer feasible. The measurements were compared with predictions based on the LHF approximation to help provide a measure of separated-flow effects. Scaling considerations of drop breakup and response were also used to interpret dense-spray properties and gain insight concerning use of the LHF approximation.

The paper begins with brief descriptions of experimental methods and the approach used for the LHF computations. Experimental results are then described, considering phase velocities, liquid breakup properties, and separated-flow parameters. The paper concludes by considering scaling of various processes in the flow. The present discussion is brief; additional details and a complete tabulation of data can be found in Ruff. ${ }^{13}$

\section{Experimental Methods}

\section{Apparatus}

The experimental apparatus was identical to past work,,$^{1,2}$ and will be described only briefly. The arrangement involved large-scale (9.5-mm injector diameter) water jets injected vertically downward in still room air. City water was supplied to the injector by a centrifugal pump with the water flow rate measured using a paddle-wheel flow meter that was calibrated by collecting water for timed intervals.

The slug and fully developed flow injectors were also identical to Ruff et al., ${ }^{1,2}$ and are described by Ruff. ${ }^{13}$ Measurements of mean and fluctuating velocities across the exit of the injectors ${ }^{13}$ showed that the slug flow injector provided relatively uniform velocities, with a streamwise turbulence intensity of roughly $1 \%$, at the jet exit, while the fully developed flow injector yielded jet exit properties that approximated fully developed turbulent pipe flow. ${ }^{14,15}$ The tests involved a mean jet velocity of $56.3 \mathrm{~m} / \mathrm{s}$, yielding other jet exit parameters as follows: water flow rate, $3.99 \mathrm{~kg} / \mathrm{s}$; Reynolds number, 534,000; Weber numbers based on gas and liquid densities, 500 and 412,000 , respectively; and Ohnesorge number, 0.00121 . These conditions are well within the atomization breakup regime and properly yielded a multiphase mixing layer that began at the exit of the injector. ${ }^{3,4}$

\section{Instrumentation}

\section{Laser Velocimetry}

The ambient air was seeded with condensed oil particles having diameters less than $1 \mu \mathrm{m}$ for $\mathrm{LV}$ measurements. The seeding particles had a flat frequency response to the gas motion up to about $30 \mathrm{kHz}$, which was adequate for present LV measurements. A large enclosure $(3 \times 3 \times 4 \mathrm{~m}$ high $)$ was constructed around the spray facility to avoid contaminating laboratory equipment with seeding particles. For an enclosure of this size, the induced counterflow velocity is less than $2 \%$ of the axial velocities in the region measured near the edge of the jets.

A phase-discriminating LV system, similar to that of Modarress et al., ${ }^{16}$ was used to avoid biasing gas-phase velocity signals with liquid velocity signals. The LV signal was obtained using the green line $(514.5 \mathrm{~nm})$ of an argon-ion laser ( $4 \mathrm{~W}$, Coherent, INNOVA 90-4) in the dual-beam forward-scatter mode. A 3.75:1 beam expander was used to minimize the dimensions of the measuring volume and to improve signalto-noise ratios. The plane of the $\mathrm{LV}$ beams was rotated to measure both streamwise and crosstream velocities. The LV was frequency shifted (40 MHz Bragg cell, TSI model $9180-$ 12) to eliminate effects of directional bias and ambiguity. The receiving optics observed the probe volume of the $L V$ at an angle of $30 \mathrm{deg}$ from the forward-scattering direction, yielding a measuring volume having a diameter of $60 \mu \mathrm{m}$ and a length of $110 \mu \mathrm{m}$. 
The phase-discrimination system involved surrounding the $\mathrm{LV}$ measuring volume with the beam from a $5-\mathrm{mW} \mathrm{HeNe}$ laser directed at an angle of $15 \mathrm{deg}$ from the LV axis, with collection optics in the forward-scattering direction also at an angle of $15 \mathrm{deg}$ from the $\mathrm{LV}$ axis. This yielded a region viewed by the discriminator that had a diameter of $0.6 \mathrm{~mm}$ and a length of $1.3 \mathrm{~mm}$, surrounding the $\mathrm{LV}$ measuring volume. Thus, droplets that could graze or cross the LV measuring volume yielded a scattering signal on the discriminator output so their velocities could be eliminated from the velocity record.

The LV scattering signal was recorded using a photomultiplier (TSI model 9160) and processed using a burst counter (TSI model $1990 \mathrm{C}$ ). The measurements involved low burst densities (one seeding particle in the measuring volume) and high data densities (time between validated velocity signals small in comparison to integral time scales); therefore, the analog output of the processor was time averaged to yield unbiased time averages. This involved low-pass filtering (Ithaco model 4213) of the output signal before it was digitized (LeCroy models $8212 \mathrm{~A} / 8$ and $8800 \mathrm{~A}$ ) and transferred to a microcomputer for processing and storage.

The performance of the phase-discriminator system was checked by measuring $L V$ data rates with and without seeding particles present. It was required that the $\mathrm{LV}$ data rate at least double for the measurements to be considered valid. Additionally, no measurement was accepted if more than $40 \%$ of the LV signal was rejected due to signals from the phase discriminator system. Evaluation of this approach, using velocities of small particles measured by double-pulse holography as an upper bound for gas velocities, indicated significant upward bias of LV gas velocity measurements by particles if these limits were exceeded.

LV signals were averaged for $2 \mathrm{~min}(128,000$ samples $)$ to provide repeatable values of mean and fluctuating gas velocities. Experimental uncertainties ( $95 \%$ confidence) are estimated to be less than $8 \%$ for mean streamwise velocities, and less than $15 \%$ for streamwise and cross-stream velocity fluctuations, largely dominated by finite sampling times. Measurements were repeatable well within these limits.

\section{Double-Pulse Holography}

LV data rates from drops alone were too high near the liquid surface so that double-pulse holography was used in this region. This approach is tedious and less accurate, due to difficulties in accumulating sufficient velocity samples and reduced particle response because only particles having diameters of roughly $5 \mu \mathrm{m}$ and greater could be measured. Nevertheless, holography provided access to portions of the multiphase mixing layer that were no longer feasible using LV.

Ruff et al. ${ }^{1}$ describe the holocamera and reconstruction systems. Velocity data were obtained over $6 \times 6 \times 4 \mathrm{~mm}$ volumes, using at least three holograms per position. The data were spatially averaged over the width of the measuring volumes, or \pm one-half the distance between adjacent radial positions, whichever was smaller. Velocity measurements were based on the motion of the centroid of the image and were correlated as a function of diameter using a linear least squares fit, considering drops having diameters less than $30 \mu \mathrm{m}$ for present estimates of gas velocities. This typically involved correlation of 50-150 individual velocity determinations. The value given by the fit at a diameter of $5 \mu \mathrm{m}$, which is the lower end of the range that could be resolved, was used as the estimate of mean gas velocities. Unfortunately, sample sizes were too small to obtain reliable estimates of velocity fluctuations.

Gradient broadening and bias errors of mean gas velocities were not significant for present conditions, yielding experimental uncertainties (95\% confidence) estimated to be less than $30 \%$, largely governed by sampling limitations (except as noted later). Experimental uncertainties ( $95 \%$ confidence) for Sauter mean diameter (SMD) and mass median diameter (MMD) were both less than $10 \%$, largely governed by sampling limitations, similar to past work. ${ }^{2}$

\section{Theoretical Methods}

Predictions of flow properties were based on the LHF approximation, neglecting evaporation of the liquid, similar to Ruff et al. ${ }^{1}$ Other major assumptions of the analysis are as follows: steady (in the mean) axisymmetric flow with no swirl; negligible kinetic energy and viscous dissipation of the mean flow; buoyancy only affects the mean flow; and equal exchange coefficients of all species and phases. Under these assumptions, the flowfield can be found using a simplified version of the conserved-scalar formalism of Lockwood and Naguib $^{17}$ but based on mass-weighted (Favre) averages, following Bilger. ${ }^{18}$ Initial conditions were based on the LV velocity measurements of jet properties at the injector exit, ${ }^{13}$ supplemented by available information on the properties of fully developed turbulent pipe flows when appropriate. ${ }^{14,15}$

Justification of the assumptions and other details of the LHF predictions can be found in Ruff et al. ${ }^{1}$ Notably, the formulation has been successfully calibrated for a variety of constant- and variable-density single-phase round jets. ${ }^{19} \mathrm{Ad}-$ ditionally, the same formation has been used successfully to estimate the structure of turbulent round air jets injected into water, which involves the same density ratio as the present flow. ${ }^{20}$ Thus, the main issue of the present evaluation is the adequacy of the LHF approximation in the near-injector region for liquid injection into gases.

\section{Results and Discussion}

\section{Phase Velocities}

\section{Mean Velocities}

Measured mean velocities in the multiphase mixing layer for slug and fully developed flow jet exit conditions are illustrated in Figs. 1 and 2 . Velocities are plotted as a function of $r / x$, the radial similarity variable for turbulent jets, to indicate the width of the flow; however, flow properties do not exhibit similarity in the $r / x$ coordinate system. Three sets of velocity measurements are shown: continuous (gas)-phase mean velocities found using the phase-discriminating LV; dispersed (drop)-phase mean velocities for 5 - $\mu$ m-diameter particles, measured using double-flash holography (which are taken to be representative of mean gas-phase velocities in the region where LV was no longer feasible); and Favre-averaged velocities of the mixture as a whole for comparison with predictions. The Favre-averaged velocities were found by summing over the sample volume $V$ containing $N$ drops, as follows:

$$
\tilde{u}=\frac{\sum_{i=1}^{N}(\pi / 6) \rho_{f} d_{p i}^{3} u_{p i}+\left(V-\sum_{i=1}^{N}(\pi / 6) d_{p i}^{3}\right) \rho_{g} \bar{u}_{g}}{\sum_{i=1}^{N}(\pi / 6) \rho_{f} d_{p i}^{3}+\left(V-\sum_{i=1}^{N}(\pi / 6) d_{p i}^{3}\right) \rho_{g}}
$$

where drop size and velocity determinations included the full range of the data reported by Ruff et al. ${ }^{1}$ Mean gas velocities in Eq. (1) were obtained from the LV measurements, if available, or from holography measurements (based on $5-\mu \mathrm{m}$-diameter drops), otherwise. The range of positions of the liquid surface, observed using holography, is indicated by the crosshatched regions in the figures. Finally, LHF predictions of Favre-averaged velocities are also illustrated on the plots. Two predictions are shown for slug flow, ignoring and allowing for boundary-layer development along the walls of the injector passage, the latter using a flow development length of $L / d=5$. These limits should bound the properties of the slug flow injector; however, differences between the two predictions are not very significant. 


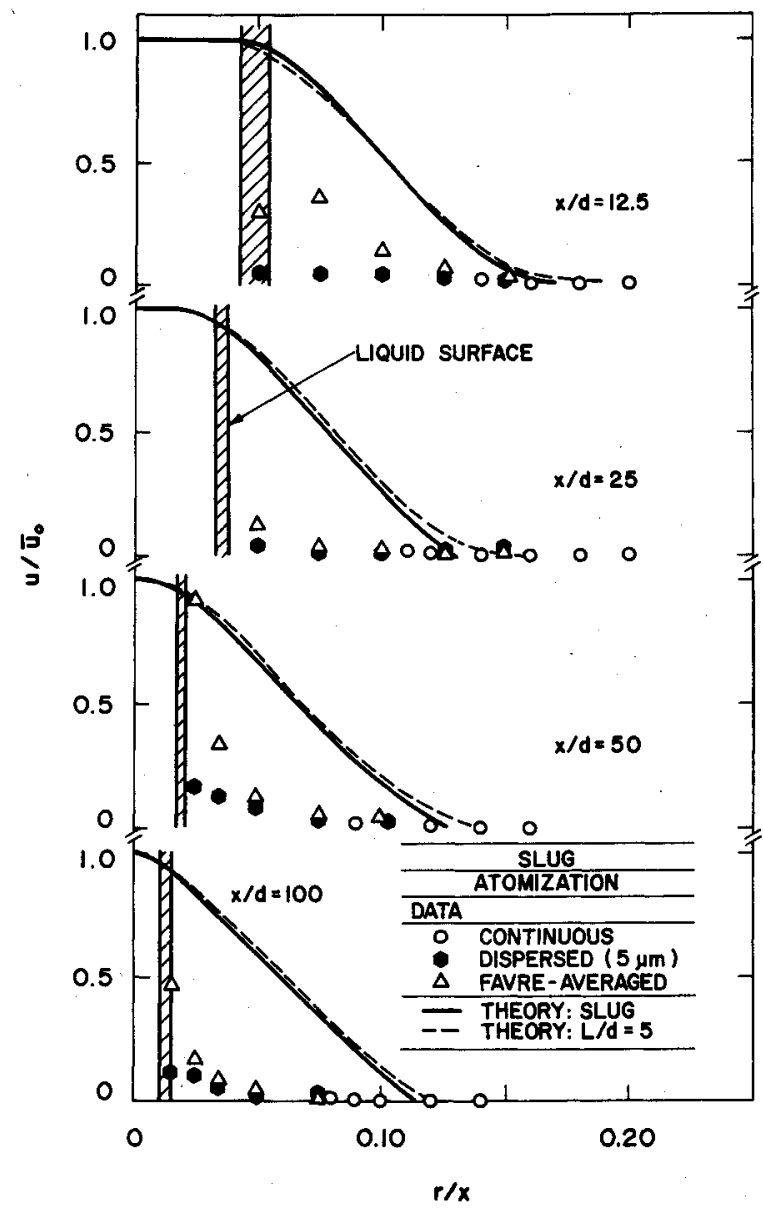

Fig. 1 Mean phase velocities for slug flow and atomization breakup.

The results for slug flow jet exit conditions (Fig. 1) exhibit reasonably good agreement between mean velocity measurements found by LV and particle tracking in the region where they overlap. Gas velocities remain quite low in the mixing layer, and only increase slightly near the liquid surface as distance from the injector exit increases. Near the injector exit, Favre-averaged velocities are significantly greater than gas velocities except near the outer edge of the mixing layer. Farther from the injector, however, differences between phase velocities are only significant near the liquid surface. Nevertheless, separated-flow effects are important throughout the mixing layer and LHF predictions of mean velocities are not satisfactory.

The results for the fully developed jet exit conditions (Fig 2) exhibit greater differences between mean velocities found by $\mathrm{LV}$ and particle tracking than for slug flow jet exit conditions. In general, velocities measured by particle tracking are biased upward from the LV results. This is felt to be the result of the breakup of larger liquid elements that are more common for fully developed flow than for slug flow, generating small drops with relatively high initial velocities. Measurement of the velocity of these drops before they relax to the gas velocity would tend to bias present velocity measurements toward higher velocities. The extent of this effect is difficult to quantify; therefore, the particle velocities of Fig. 2 are at best representative of an upper bound on gas velocities. Similar to slug flow, gas velocities remain relatively low throughout the mixing layer, Favre-averaged velocities are generally significantly greater than gas velocities indicating significant effects of separated flow, and LHF predictions are not very satisfactory as a result.

\section{Gas Velocity Fluctuations}

Measured and predicted gas velocity fluctuations are illustrated in Figs. 3 and 4 for slug and fully developed flow jet

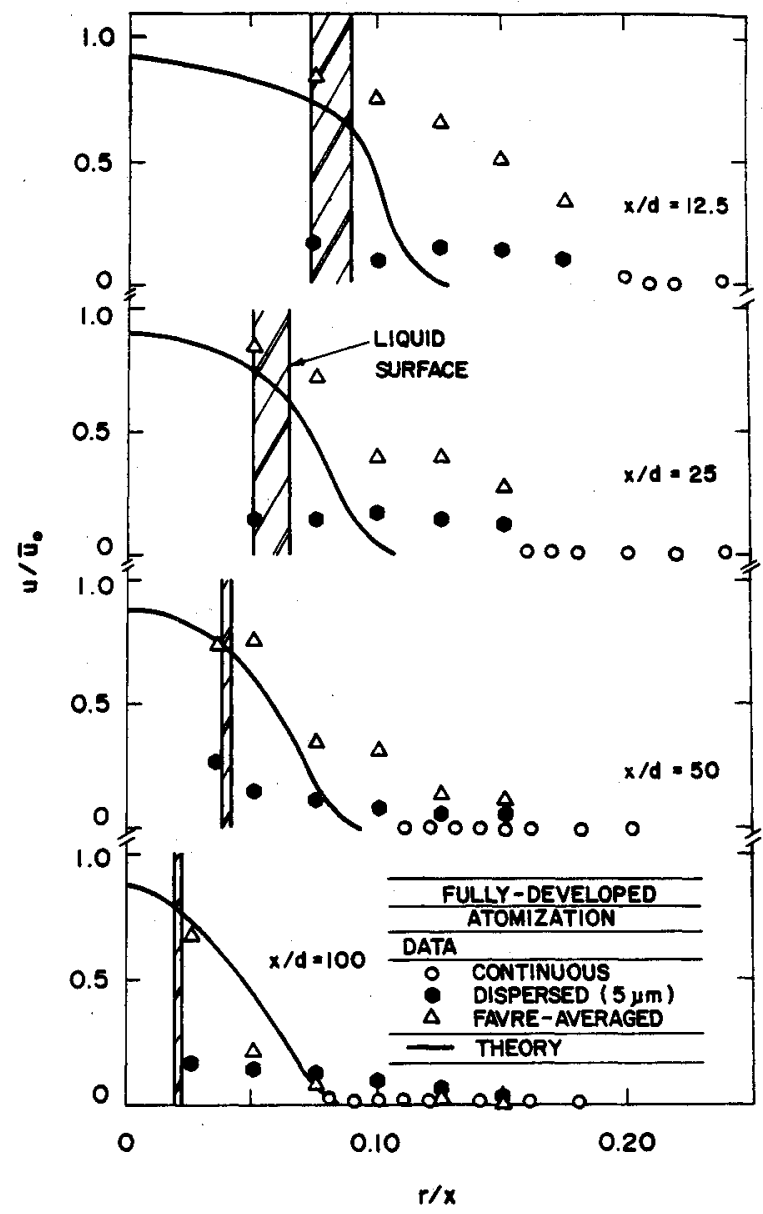

Fig. 2 Mean phase velocities for slug flow and atomization breakup.

exit conditions. The measurements are limited to streamwise and radial velocity fluctuations found by $L V$, which generally only covers the outer half of the mixing layer. Predictions are based on the isotropic approximation, $\bar{u}^{\prime \prime 2}=\bar{v}^{\prime \prime 2}=2 k / 3$, and are Favre averages under the LHF assumption. Measured velocity fluctuations are nearly isotropic near the outer edge of the mixing layer, which is similar to the behavior of singlephase jets. Streamwise velocity fluctuations are substantially greater than radial velocity fluctuations, however, as the spray becomes dense (this is particularly evident for $x / d=12.5$ ). Such behavior is typical of dense sprays in regions where relative velocities are significant. ${ }^{5,21,22}$ Other than indicating that velocity fluctuations should increase in the dense portions of the mixing layer, in a qualitative way, the LHF predictions of velocity fluctuations are not very useful.

\section{Liquid Breakup}

\section{Drop-Size Distributions}

Table 1 is a summary of drop-size properties for nonturbulent and turbulent jet exit conditions. These properties were relatively independent of position along the liquid surface, and over the flow cross section if the region near the liquid surface was excluded. Thus, SMD and MMD/SMD ratio have been grouped accordingly in Table 1 with properties over the cross section represented by results at $x / d=100$. Gas velocities along the liquid surface varied irregularly due to experimental uncertainties so that only the range of values is summarized in Table 1. Velocities in the liquid core remain close to jet exit velocities, ca. $56 \mathrm{~m} / \mathrm{s}^{\mathrm{I}}$

For each jet exit turbulence level, SMD along the liquid surface is substantially greater than over the cross section, providing evidence for significant effects of secondary breakup. Additionally, liquid turbulence substantially increased drop 


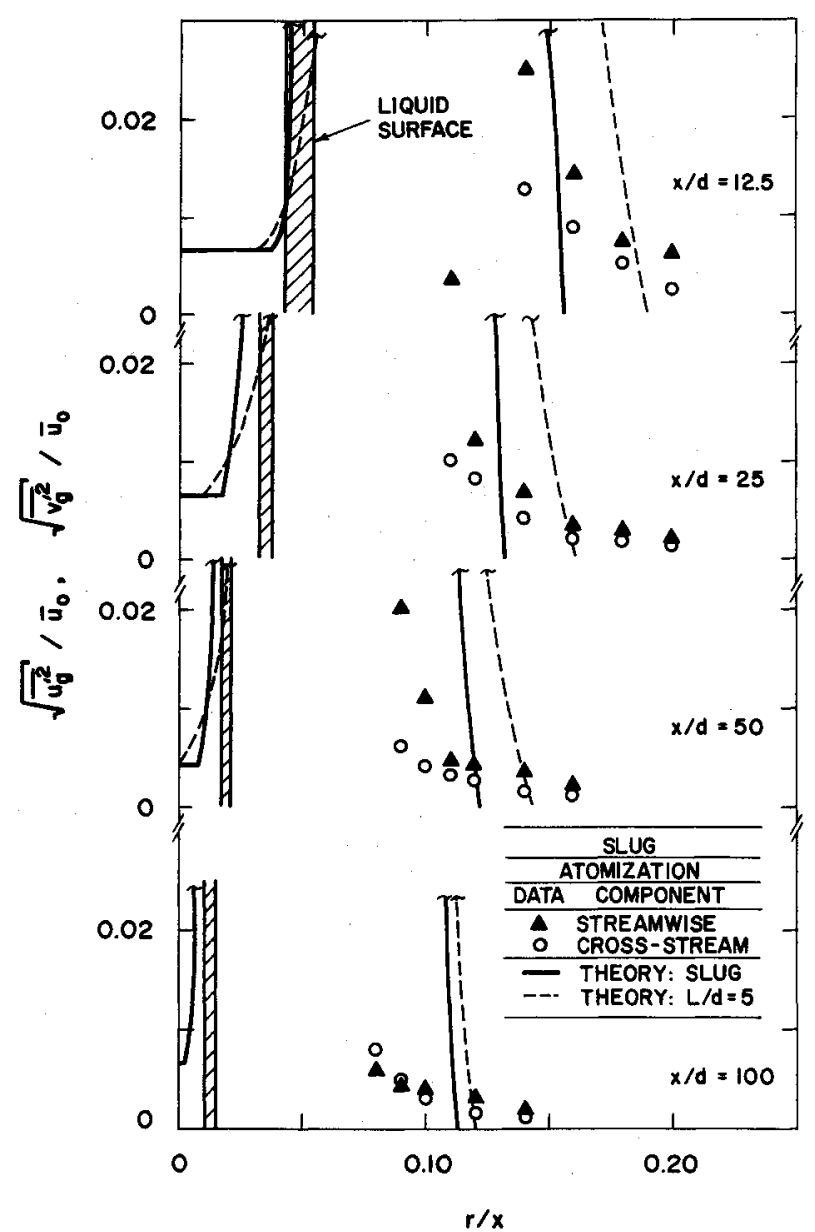

Fig. 3 Gas-phase velocity fluctuations for slug flow and atomization breakup.

sizes everywhere in comparison to nonturbulent conditions: roughly $7: 1$ after primary breakup at the surface, and roughly 2:1 over the cross section. In view of the larger drop sizes for a turbulent liquid, it is somewhat surprising that this flow mixes fastest (cf. Figs. 1 and 2). This behavior is due to faster primary breakup for the turbulent liquid, ${ }^{1}$ perhaps with enhanced radial drop velocities due to radial velocity fluctuations in the liquid.

In spite of effects of turbulence on primary breakup, as well as secondary breakup, the MMD/SMD ratio is roughly 1.2 for all conditions in Table 1 . This value agrees with observations for a wide variety of nonevaporating dilute sprays. ${ }^{23}$ This motivated examination of whether the drop-size distributions in the dense sprays satisfy Simmons' universal rootnormal drop-size distribution function. ${ }^{23}$ These results are illustrated in Fig. 5, considering every position measured for both nonturbulent and turbulent jet exit conditions. It is evident that the root-normal distribution with MMD/SMD = 1.2 provides a reasonably good fit of all the measurements. Notably, except for $x / d=12.5$, where effects of flow development are strongest, individual evaluations of the MMD/ SMD ratio yield a value of roughly 1.2 as well. The correlation of Fig. 5 is very helpful because the entire drop-size distribution can be related in a simple manner to SMD. ${ }^{23}$ However, an explanation of this behavior and more evidence that it is generally suitable for dense sprays is needed.

\section{Nonturbulent Primary Breakup}

Present results indicate different breakup mechanisms for nonturbulent and turbulent liquids at otherwise identical conditions in the atomization breakup regime with low $O h$. It is generally agreed that atomization breakup at low $O h$ involves a stripping mechanism for nonturbulent liquids. ${ }^{2,3,5}$ Among

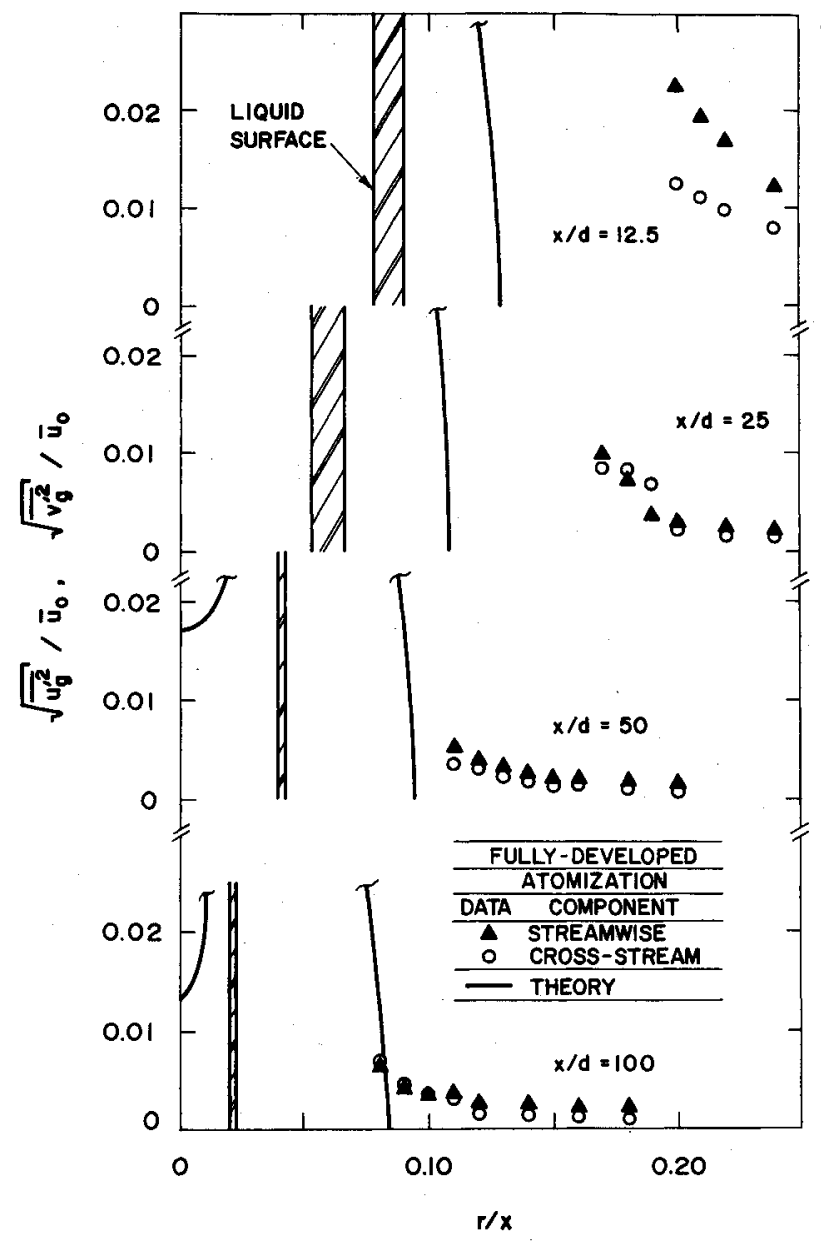

Fig. 4 Gas-phase velocity fluctuations for fully developed flow and atomization breakup.

Table 1 Drop-size properties ${ }^{a}$

\begin{tabular}{|c|c|c|c|c|c|}
\hline \multirow[b]{2}{*}{ Flow } & \multicolumn{3}{|c|}{$\begin{array}{l}\text { Liquid surface, } 12.5 \\
\leq x / d \leq 100\end{array}$} & \multicolumn{2}{|c|}{$\begin{array}{c}\text { Cross section, } \\
x / d=100\end{array}$} \\
\hline & $\begin{array}{l}\tilde{u}_{g s} \\
\mathrm{~m} / \mathrm{s}^{\mathrm{b}}\end{array}$ & $\underset{\mu \mathrm{m}}{\mathrm{SMD}}$ & $\begin{array}{l}\mathrm{MMD} / \\
\mathrm{SMD}\end{array}$ & $\begin{array}{c}\mathrm{SMD} \\
\mu \mathrm{m}\end{array}$ & $\begin{array}{l}\text { MMD/ } \\
\text { SMD }\end{array}$ \\
\hline Slug & $3-9$ & 17 & 1.21 & 95 & 1.18 \\
\hline Fully developed & $8-15$ & 1330 & 1.20 & 220 & 1.22 \\
\hline
\end{tabular}

aSMD and MMD are based on equivalent spherical drops as described in Ruff et al. ${ }^{2}$

${ }^{\mathrm{b}} \mathrm{Gas}$ velocity estimated from $5-\mu \mathrm{m}$-diam drops near surface.

others, Levich ${ }^{24}$ has proposed a formula to estimate drop sizes due to primary breakup for these conditions based on Taylor's ${ }^{25}$ theory of aerodynamically induced growth of surface waves, assuming that the average diameter of drops formed at the surface is proportional to the wavelength of the unstable surface waves having the highest growth rates. Using SMD to represent the average drop diameter then yields

$$
\mathrm{SMD}=6 \pi C_{B} \sigma /\left[\rho_{g}\left(\bar{u}_{f s}-\bar{u}_{g s}\right)^{2}\right]
$$

where $C_{B}$ is a constant of proportionality near unity.

For present conditions, $\bar{u}_{f s}$ is roughly equal to $u_{0}=56.3$ $\mathrm{m} / \mathrm{s}$, based on LHF computations, as well as measurements of the velocity of protuberances from the liquid surface. Then, matching the primary breakup SMD in Table 1, using an average value of $\bar{u}_{g s}$, yields $C_{B}=0.4$. Since $C_{B}$ is expected to be on the order of unity, the aerodynamic primary breakup correlation of Eq. (2) appears to be reasonable for nonturbulent conditions within dense sprays. However, additional evaluation of the expression for other test conditions is clearly needed. 


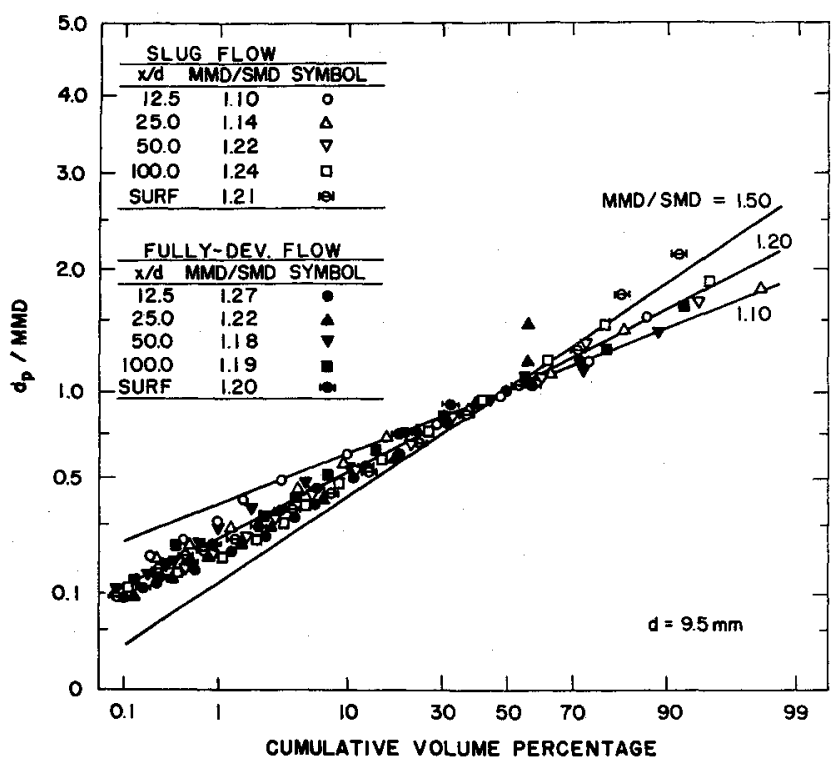

Fig. 5 Drop-size distributions at liquid surface and across mixing layer for slug and fully developed flow and atomization breakup.

\section{Turbulent Primary Breakup}

The much larger drop sizes after primary breakup for turbulent jet exit conditions are best explained by a turbulent breakup mechanism with relatively small aerodynamic effects. Lee and Spencer ${ }^{26}$ report early observations of the turbulent breakup mechanism, based on tests at low ambient pressures where aerodynamic effects are negligible. Reitz ${ }^{27}$ and Reitz and $\mathrm{Bracco}^{28}$ also identify breakup induced by liquid-phase turbulence as one of the possible mechanisms contributing to the atomization process. This mechanism involves turbulent distortion of the liquid surface to form drops, with surface tension acting to stabilize the distortion, so that maximum drop sizes should be comparable to the radial integral scale of the liquid turbulence. Taking the SMD to be representative of maximum drop sizes, this implies

$$
\operatorname{SMD} / \Lambda=C_{t}^{\prime}
$$

where $C_{t}^{\prime}$ is a constant on the order of unity. Laufer ${ }^{14,29}$ finds $\Lambda / d=0.10-0.15$ for turbulent pipe flow while properties within the liquid core do not vary significantly from jet exit conditions based on the LHF computations. ${ }^{1}$ Then, based on the SMD measurements in Table 1 for turbulent jet exit conditions, Eq. (3) yields $C_{t}^{\prime}=0.9-1.3$, with the range resulting from Laufer's ${ }^{29}$ range of $\Lambda / d$. Additionally, the ratio of streamwise to cross-stream integral scales for turbulent pipe flow is roughly $4: 1,{ }^{29}$ which is comparable to the mass-averaged ellipticity (ratio of maximum to minimum liquid element dimensions) observed for turbulent breakup conditions near the liquid surface. ${ }^{1}$ Thus, the turbulent breakup mechanism provides a plausible description of the large-scale features of the sprays after primary breakup.

Additional evidence supporting the turbulent breakup mechanism can be found by considering the criterion for this mechanism to be active. This can be estimated, similar to transition from the drip to the jetting regime of liquid jets in gases, ${ }^{30}$ by equating the momentum available from turbulent fluctuations of scale $d_{p}$ to the surface tension forces resisting the formation of a drop of similar size. Using the relationship between velocity fluctuations and eddy size within the inertial region of the turbulence spectrum, ${ }^{29}$ the expression for the range of drop sizes that can be formed by the turbulent primary breakup mechanism is

$$
\rho_{f} \Lambda \bar{v}_{f}^{2}\left(d_{p} / \Lambda\right)^{5 / 3} / \sigma \geq 6 C_{t}
$$

where $C_{t}$ is a constant on the order of unity. Taking $C_{t}=1$, Eq. (4) implies that the minimum drop diameter resulting from turbulent breakup for the conditions of Table 1 is ca. $250 \mu \mathrm{m}$, which is in good agreement with observations. In contrast, Eq. (4) is not satisfied even when $d_{p}=\Lambda$ for the first wind-induced test condition for the present sprays. ${ }^{1,2}$ This also corresponds to observations because the liquid surface only was roughened by turbulent distortion for this test condition and no drop breakup occurred. Thus, the turbulent breakup mechanism is consistent with present observations and deserves additional study over a wider range of test conditions.

Equations (3) and (4) also help to put observations of effects of liquid turbulence on primary breakup for the present largescale sprays into perspective. For example, small injector diameters imply relatively small maximum drop sizes from this mechanism, while the aerodynamic breakup correlation, Eq. (2), is essentially independent of injector diameter (as long as $O h$ is sufficiently low. Thus, aerodynamic breakup is likely to be more dominant for conventional sized injectors, because it yields larger drops than turbulent breakup, even when jet exit conditions are fully turbulent. Additionally, most practical injectors have small length-to-diameter ratios so that liquid turbulence is not highly developed even when jet Reynolds numbers are large. Based on these considerations, turbulent primary breakup is probably not a major feature of most practical sprays, as suggested by Reitz ${ }^{27}$ and Reitz and Bracco. ${ }^{28}$

\section{Secondary Breakup}

Criteria for secondary breakup are frequently stated in terms of a drop Weber number, defined as follows:

$$
W e_{p g}=\rho_{g} d_{p}\left(u_{p}-u_{g}\right)^{2} / \sigma
$$

One criterion, discussed by Clift et al. ${ }^{30}$ is based on water drops accelerated by shock waves, yielding:

$$
W e_{p g}>W e_{p g}^{*}=6.5
$$

Another criterion, discussed by Pruppacker and Klett, ${ }^{31}$ was obtained for liquid drops falling in still air:

$$
W e_{p g}>W e_{p g}^{*}=8 / C_{D}^{*}
$$

For present test conditions, drop drag coefficients are in the range of $0.8-1.2$ for the largest drops, based on the standard drag coefficient for spheres ${ }^{5}$; therefore, Eqs. (6) and (7) yield similar values of $W e_{p g}^{*}$.

In order to quantify the potential for secondary drop breakup in the mixing layer, mass-weighted Weber numbers were computed as follows:

$$
\tilde{W} e_{g}=\sum_{i=1}^{N}\left(\rho_{g} d_{p}\left(u_{p}-\bar{u}_{g}\right)^{2} / \sigma\right)_{i} d_{p i}^{3} / \sum_{i=1}^{N} d_{p i}^{3}
$$

given $N$ drop measurements at a point. The resulting distributions of $W e_{g}$ for the sprays having slug and fully developed jet exit conditions are plotted in Fig. 6. The values of $\tilde{W} e_{g}$ are greatest near the liquid surface and decrease monotonically with increasing distance from the surface. In general, values of $\tilde{W} e_{g}$ near the liquid surface exceed $W e_{p g}^{*}$ for breakup from Eqs. (6) and (7), supporting significant effects of secondary breakup in the mixing layer. The larger liquid elements ejected from the surface when the liquid is turbulent imply greater effects of secondary breakup for these conditions.

Primary followed by secondary breakup is likely to be a general mechanism for dense sprays. For example, the value of $W e_{p g}$ for a drop having a diameter equal to the SMD is roughly 8 for aerodynamic primary breakup from Eq. (2). Thus, drops having diameters of the order of the SMD and 


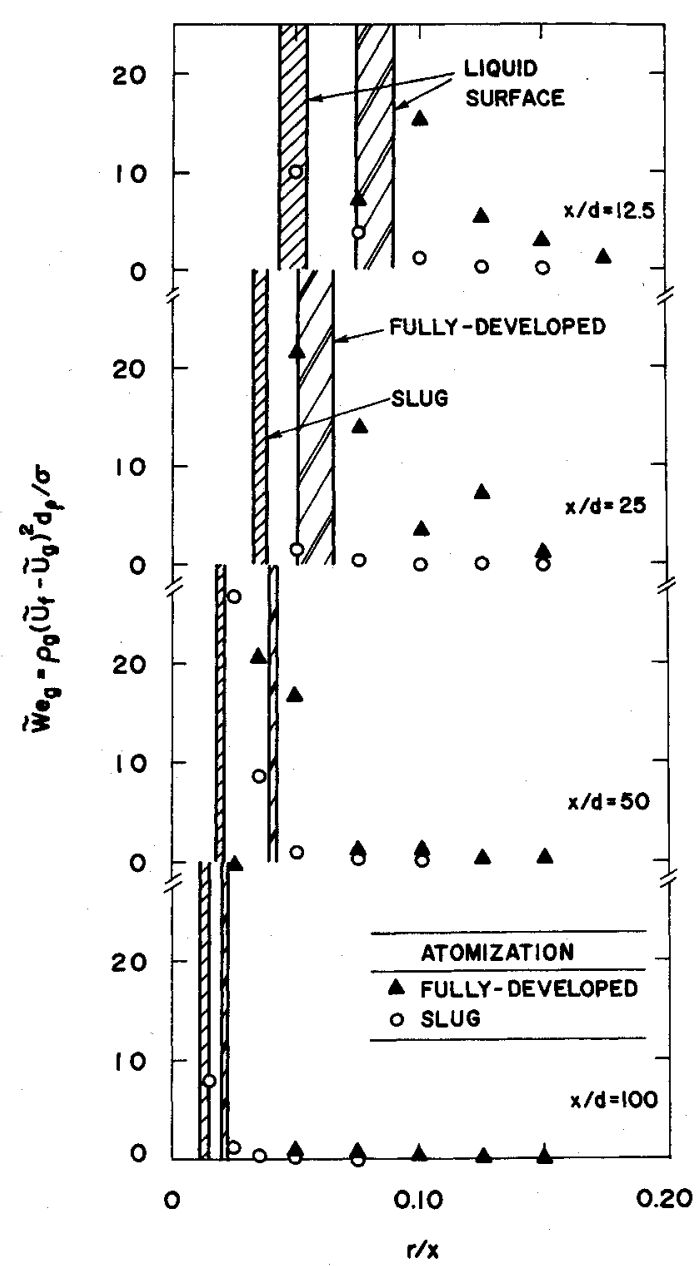

Fig. 6 Favre-averaged drop Weber numbers for slug and fully developed flow and atomization breakup.

larger, which comprise more than half the mass of the liquid in the spray for MMD/SMD $=1.2$, have Weber numbers that exceed $W e_{p g}^{*}$ from Eqs. (6) and (7). Naturally, the turbulent breakup mechanism, if active, yields even larger values of SMD and a greater propensity for secondary breakup. These observations, as well as the relatively small liquid volume fractions within the multiphase mixing layer, ${ }^{1}$ suggest that secondary breakup rather than drop collisions is a dominant feature of dense sprays.

\section{Separated Flow Effects}

The results illustrated in Figs. 1 and 2 are somewhat misleading because small velocities give the impression that separated flow effects are small near the edge of the flow. This is not the case, which can be seen from the plots of Favreaveraged separated-flow factor, defined as follows:

$$
S=\left(\tilde{u}_{f}-\tilde{u}_{g}\right) / \tilde{u}_{f}
$$

Distributions of $S$ are illustrated in Fig. 7 for both slug and fully developed jet exit conditions. Values of $S$ approaching unity are observed throughout the mixing layer near the jet exit, and near the liquid surface for all streamwise positions. Values of $S$ near the outer edge of the flow tend to decrease with increasing distance from the injector, however, approaching values in the range $0.1-0.2$ for fully developed jet exit conditions at $x / d=100$. In spite of the presence of larger drops near the liquid surface, values of $S$ are generally lower for fully developed than slug flow jet exit conditions.

The trends seen in Fig. 7 result from both the generation of drops at the liquid surface and momentum exchange between the phases in the mixing layer. Velocities within the liquid core remain near liquid injection velocities so that newly

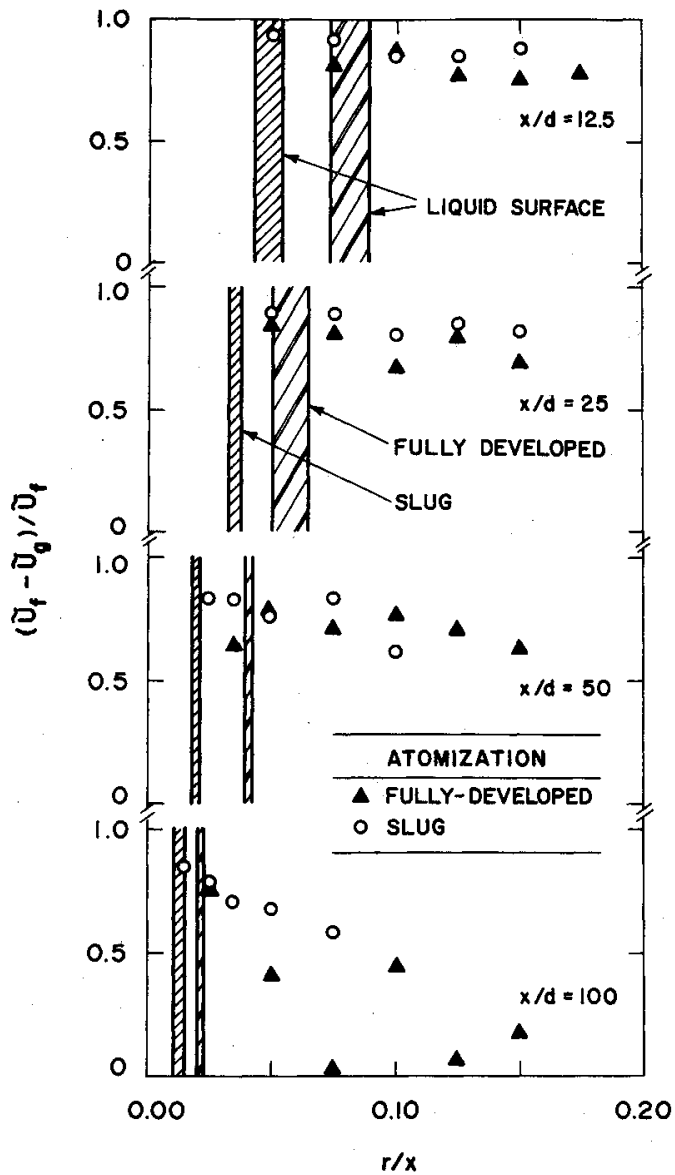

Fig. 7 Favre-averaged separated-flow factor for slug and fully developed flow and atomization breakup.

formed drops near the injector exit and along the liquid surface generally have velocities that are significantly greater than gas velocities, yielding relatively large values of $S$. Drops near the outer edge of the flow, however, must have had significant residence times in the gas so that turbulent dispersion can transport them to this region. Therefore, drops near the edge of the flow tend to relax toward gas velocities, particularly when residence times are long (at large $x / d$ ). Additionally, fully developed jet exit conditions increase drop concentrations in the mixing layer in comparison to slug flow exit conditions, causing greater acceleration of the gas within the mixing layer. This compensates for the higher velocities associated with larger drops produced by fully developed exit conditions and tends to reduce $S$. Nevertheless, the values of $S$ on the order of unity throughout most of the mixing layer, separated-flow effects are clearly important, which explains why predictions based on the LHF approximation are poor for present test conditions.

\section{Scaling}

\section{Characteristic Times}

Characteristic times are considered in the following to evaluate secondary breakup and effects of separated flow, and to help relate present observations for large sprays to practical pressure-atomized sprays. Characteristic times of interest are flow residence, liquid core residence, drop response, and drop breakup times. The largest drops are of interest, which have velocities of roughly $u_{0}$; therefore, an appropriate flow residence time is

$$
\tau_{f}=x / u_{0}
$$

where $x$ is to be interpreted as the distance from the position where the drop entered the mixing layer after primary breakup. 
It is also of interest to assess response over the length of the liquid core as a measure of the length of the dense-spray region. Taylor's ${ }^{25}$ aerodynamic breakup analysis yields the following expression for the length of the liquid core:

$$
L_{c} / d=C_{c}\left(\rho_{f} / \rho_{g}\right)^{1 / 2}
$$

Chehroudi et al. ${ }^{8}$ find $C_{c}$ in the range $7-16$ for the contiguous length of liquid core for pressure atomized round jets in still gases within the atomization breakup regime. Taking the characteristic velocity of large drops to be $u_{0}$, the characteristic residence time of the liquid core becomes:

$$
\tau_{c}=L_{e} / u_{0}=C_{c}\left(d / u_{0}\right)\left(\rho_{f} / \rho_{g}\right)^{1 / 2}
$$

The largest drops have the longest characteristic response times. The results illustrated in Fig. 6 suggest that the largest drops have Weber numbers comparable to $W e_{p g}^{*}$ from Eq. (6) for both laminar and turbulent liquid conditions, and this condition will be used as a measure of maximum drop sizes. This yields a critical drop size of

$$
d_{p}^{*}=\sigma W e_{p g}^{*} /\left(\rho_{g} u_{0}^{2}\right)
$$

assuming that relative velocities are comparable to $u_{0}$ based on present measurements for large drops. ${ }^{1} \mathrm{~A}$ characteristic response time for drops can be defined in terms of their rate of deceleration, as follows:

$$
\tau_{p}=-\left(\mathrm{d} u_{p} / \mathrm{d} x\right)^{-1}=-u_{p} /\left(\mathrm{d} u_{p} / \mathrm{d} t\right)
$$

Virtual mass and Basset history forces generally can be ignored when computing drop motion in sprays because $\rho_{f} / \rho_{g}$ $>1$, while pressures within the mixing layer are constant; therefore, conservation of momentum for a drop yields ${ }^{5}$ :

$$
\mathrm{d} u_{p} / \mathrm{d} t=-3 \rho_{g} C_{D}\left(u_{p}-u_{g}\right)^{2} /\left(4 \rho_{f} d_{p}\right)
$$

Streamwise velocities have been assumed to be large in comparison to cross-stream velocities to obtain Eq. (15), which is justified due to the boundary-layer character of the mixing layer. Substituting Eq. (15) into Eq. (14), assuming $u_{g} \ll u_{p}$ $\approx u_{0}$ as before, and using Eq. (13) to identify the critical drop size, then yields:

$$
\tau_{p}=4 W e_{p g}^{*} \rho_{f} \sigma /\left(3 C_{D}^{*} \rho_{g}^{2} u_{o}^{3}\right)
$$

Because $C_{D}$ does not vary appreciably for large drops in sprays, ${ }^{5}$ Eq. (16) shows that increased gas densities and jet exit velocities reduce drop response times, both due to effects of breakup which reduces drop sizes and the relative increase of drag in comparison to inertia of the drops for a particular size [see Eq. (15)].

Finally, the characteristic time required for secondary breakup is an important feature of dense sprays. As noted earlier, drop Weber numbers are not much greater than the breakup limit, so that deformation and stripping breakup are the main mechanisms of secondary breakup..$^{32}$ Therefore, adopting the correlation of Ranger and Nicholls ${ }^{33}$ for stripping breakup yields

$$
\tau_{b}=C_{b} d_{p}\left(\rho_{f} / \rho_{g}\right)^{1 / 2} /\left(u_{p}-u_{g}\right)
$$

where the empirical factor $C_{b} \approx 4$. Taking $d_{p}=d_{p}^{*}$ and $u_{g}$ $\ll u_{p} \approx u_{0}$, as before, Eq. (17) becomes:

$$
\tau_{b}=C_{b} W e_{p g}^{*} \sigma\left(\rho_{f} / \rho_{g}\right)^{3 / 2} /\left(\rho_{f} u_{0}^{3}\right)
$$

\section{Characteristic Time Ratios}

A variety of characteristic time ratios can be formed to highlight various properties of dense sprays. Forming the ratio $\tau_{b} / \tau_{p}$ from Eqs. (16) and (18) yields:

$$
\tau_{b} / \tau_{p}=\left(3 C_{b} C_{D}^{*} / 4\right)\left(\rho_{g} / \rho_{f}\right)^{1 / 2}
$$

Small values of $\tau_{b} / \tau_{c}$ imply that secondary breakup times are small in comparison to the time required for drops to relax to the local gas velocity. Taking $C_{D}^{*}=1$, as before, implies that $\tau_{b} / \tau_{p}$ is ca. 0.1 for present test conditions so that secondary breakup is relatively fast, which agrees with observations that the largest drops were close to the liquid surface. Under these conditions, the outcome of secondary breakup has the greatest effect on dense-spray properties. At high pressures, however, $\tau_{b} / \tau_{p} \approx 1$ and breakup extends over distances required for drops to approach gas velocities; then, drop breakup must be treated like a finite rate process, similar to effects of drag and vaporization on drop motion.

Another perspective on secondary breakup can be found from the ratio of Eqs. (12) and (18)

$$
\tau_{b} / \tau_{c}=C_{b} W e_{p g}^{*} /\left(C_{c} W e_{g}\right)
$$

which is the ratio of secondary breakup to liquid core residence time. For present test conditions, $\tau_{b} / \tau_{c}$ ca. 0.005 , so that breakup times are short in comparison to residence times in the mixing layer. This also agrees with observations that the large drops resulting from primary breakup were associated with the region near the liquid surface. However, this is not always the case. For example, transition from wind-induced to atomization breakup requires $W e_{g}>W e_{g}^{*}=13-$ $40 .^{2,3}$ Near the transition limit, $\tau_{b} / \tau_{c}$ is in the range $0.1-0.3$, which implies that secondary breakup proceeds rather slowly within the mixing layer (particularly because new drops are formed all along the liquid core). Under these conditions, rates of secondary breakup should control processes in dense sprays, somewhat like drop drag and vaporization tends to control processes in dilute sprays. from

Insight concerning effects of separated flow can be obtained

$$
\tau_{f} / \tau_{p}=\left(3 C_{D}^{*} / 4 W e_{p g}^{*}\right)\left(\rho_{g} / \rho_{f}\right)^{2}\left(\rho_{f} x u_{0}^{2} / \sigma\right)
$$

where large values of $\tau_{f} / \tau_{p}$ correspond to regions of the flow where effects of separated flow are small. For present flows, drops continue to be formed at the liquid surface throughout the region where measurements were made. Therefore, characteristic distances, $x$, and the corresponding values of $\tau_{f} / \tau_{p}$ remain small, which is consistent with the large values of $\left(\tilde{u}_{f}\right.$ $\left.-\tilde{u}_{g}\right) / \tilde{u}_{f}$ seen in Fig. 7 in this region. In contrast, drops near the edge of the flow originate near the jet exit. Then, estimating the empirical parameters in Eq. (21) as before, $\tau_{f} / \tau_{p}$ varies in the range $0.5-4$ as $x / d$ varies in the range $12.5-100$. This implies significant effects of separated flow near the jet exit, with separated-flow effects decreasing near the edge of the flow at larger streamwise distances, which is also consistent with the results illustrated in Fig. 7.

For a round jet, most of the liquid enters the mixing layer near the jet exit, and large values of the ratio

$$
\tau_{c} / \tau_{p}=\left(3 C_{c} C_{D}^{*} / 4 W e_{p g}^{*}\right)\left(\rho_{g} / \rho_{f}\right)^{3 / 2} W e_{f}
$$

implies that separated-flow effects are small within the densespray region. The effect of the density ratio $\rho_{g} / \rho_{f}$ is somewhat reduced for $\tau_{c} / \tau_{p}$ in comparison to $\tau_{f} / \tau_{p}$, because the length of the liquid core is reduced as this density ratio increases through Eq. (11). For present test conditions, $L_{c} / d$ is in the range 200-460 from Eq. (11) while $\tau_{c} / \tau_{p}$ is in the range 1125 from Eq. (22). Thus, the values of $\tau_{c} / \tau_{p}$ imply modest effects of separated flow over the length of the liquid core, although separated-flow effects are still significant for the 
relatively low range of $x / d(x / d \leq 100)$ where present measurements were made. More generally, Eq. (22) implies that large-diameter jets, like those of the present experiments, have reduced effects of separated flow because of their large $W e_{f}$ in comparison to practical sprays having similar jet exit velocities. Thus, the LHF limit is approached at large $W e_{f}$, with this limit approached more rapidly as $\rho_{g} / \rho_{f}$ increases, as long as $O h$ is sufficiently small. Additional measurements, however, are needed to confirm the trends suggested by Eqs. (19-22).

\section{Conclusions}

The multiphase mixing layer in the near-injector region of large-scale pressure-atomized sprays was investigated, considering both nonturbulent slug and fully developed turbulent pipe flow at the jet exit. The major conclusions of the study are as follows:

1. Present measurements, combined with the earlier results of Ruff et al. ${ }^{1}$ for the same flows, tend to support the classical view of atomization: a surprisingly dilute multiphase mixing layer (liquid volume fractions less than $1 \%$ ) dominated by secondary breakup and separated-flow processes, while effects of collisions appear to be small.

2. Use of the LHF approximation was not effective for estimating the properties of the multiphase mixing layers for present test conditions, because large drops are continuously formed along the liquid surface and require significant times to relax to gas velocities. Characteristic time considerations suggest that locally homogenous flow is approached at large $W e_{f}$, with this limit approached more rapidly as $\rho_{g} / \rho_{f}$ increases.

3 . For nonturbulent jet exit conditions, the SMD for primary breakup agreed with aerodynamic breakup theory, ${ }^{24}$ with $C_{B}=0.4$. For turbulent jet exit conditions, SMD for primary breakup was explainable by turbulent breakup theory with the SMD roughly equal to the radial integral scale of the liquid turbulence.

4. Drop-size distributions after aerodynamic primary breakup, turbulent primary breakup, and secondary breakup all exhibited MMD/SMD ratios of roughly 1.2 and agreed reasonably well with Simmons's root-normal drop-size distribution. ${ }^{23}$ This is a useful simplification because the entire drop-size distribution is known if a single moment, like SMD, can be found.

5. An expression for the range of drop sizes that can be formed from the turbulent primary breakup mechanism was developed and indicated the dependence of drop size on the integral scale and intensity of liquid-phase turbulence. The expression was generally supported by present measurements. However, the importance of this breakup mechanism for practical injectors having small diameters is questionable.

6. Based on present measurements, more than half of the mass of the spray is contained in drops that are unstable to secondary breakup after aerodynamic breakup, while turbulent primary breakup yields larger drops that have a greater propensity for secondary breakup. Thus, secondary breakup appears to be an intrinsic process of dense sprays in the atomization spray breakup regime.

The conclusions are based on limited measurement for liquid jets having large diameters which have relatively slow flow development rates in comparison to practical injectors and are only provisional pending additional experimental evaluation.

\section{Acknowledgments}

This research was sponsored by the Air Force Office of Scientific Research Grants AFOSR 85-0244 and 89-0516, and by the Office of Naval Research, Grant N00014-89-J-1199. The U.S. Government is authorized to reproduce and distribute copies for governmental purposes notwithstanding any copyright notation thereon.

\section{References}

${ }^{1}$ Ruff, G. A., Bernal, L. P., and Faeth, G. M., "Structure of the Near-Injector Region of Non-Evaporating Pressure-Atomized Sprays," Journal of Propulsion and Power, Vol. 7, No. 2, 1991, pp. 221-230.

${ }^{2}$ Ruff, G. A., Sagar, A. D., and Faeth, G. M., "Structure and Mixing Properties of Pressure-Atomized Sprays," AIAA Journal, Vol. 27, No. 7, 1989, pp. 901-908.

${ }^{3}$ Ranz, W. E., "Some Experiments on Orifice Sprays," Canadian Journal of Chemical Engineering, Vol. 36, Aug. 1958, pp. 175-181.

${ }^{4}$ Miesse, C. C., "Correlation of Experimental Data on Disintegration of Liquid Jets," Industrial and Engineering Chemistry, Vol. 47, Sept. 1955, pp. 1690-1697.

${ }^{5}$ Faeth, G. M., "Mixing, Transport and Combustion in Sprays," Progress in Energy Combustion Science, Vol. 13, 1987, pp. 293-345.

'Phinney, R. E., "The Breakup of a Turbulent Liquid Jet in a Gaseous Atmosphere," Journal of Fluid Mechanics, Vol. 60, Oct. 1973, pp. 689-701.

${ }^{7}$ Hiroyasu, H., Shimizu, M., and Arai, M., "The Breakup of a High Speed Jet in a High Pressure Gaseous Environment," ICLASS82, Univ. of Wisconsin, Madison, WI, 1982.

${ }^{8}$ Chehroudi, B., Onuma, Y., Chen, S.-H., and Bracco, F. V., "On the Intact Core of Full-Cone Sprays," SAE Paper 850126, 1985.

${ }^{9} \mathrm{Wu}$, K.-J., Su, C.-C., Steinberger, R. L., Santavicca, D. A., and Bracco, F. V., "Measurements of the Spray Angle of Atomizing Jets," Journal of Fluids Engineering, Vol. 105, Dec. 1983, pp. 406415.

${ }^{10} \mathrm{Wu}$, K.-J., Coghe, A., Santavicca, D. A., and Bracco, F. V., "LDV Measurements of Drop Velocity in Diesel-Type Sprays," $A I A A$ Journal, Vol. 22, No. 9, 1984, pp. 1263-1270.

${ }^{11}$ Mao, C.-P., Wakamatsu, Y., and Faeth, G. M., "A Simplified Model of High Pressure Spray Combustion," Eighteenth Symposium (International) on Combustion, The Combustion Institute, Pittsburgh, 1980, pp. 337-347.

${ }^{12}$ Bracco, F. V., "Structure of High-Speed Full-Cone Sprays," Recent Advances in Gas Dynamics, edited by C. Casci, Plenum Publishing Corp., New York, 1983.

${ }^{13}$ Ruff, G. A., "Structure and Mixing Properties of the Near-Injector Region of Nonevaporating Pressure-Atomized Sprays," Ph.D. Thesis, Univ. of Michigan, Ann Arbor, MI, 1990.

${ }^{14}$ Hinze, J. O., Turbulence, 2nd ed., McGraw-Hill, New York, 1975, pp. 427, 724-734.

${ }^{15}$ Schlichting, H., Boundary Layer Theory, 7th ed., McGraw-Hill, New York, 1979, p. 599

${ }^{16}$ Modarress, D., Tan, H., and Elgobashi, S., "Two-Component LDA Measurements in a Two-Phase Turbulent Jet," AIAA Journal, Vol. 22, No. 5, 1984, pp. 624-630.

${ }^{17}$ Lockwood, F. C., and Naguib, A. S., "The Prediction of Fluctuations in the Properties of Free, Round-Jet Turbulent Diffusion Flames," Combustion and Flame, Vol. 24, Feb. 1975, pp. 109-124.

${ }^{18}$ Bilger, R. W., "Turbulent Jet Diffusion Flames," Progress in Energy Combustion Science, Vol. 1, 1976, pp. 87-109.

${ }^{19}$ Jeng, S.-M., and Faeth, G. M., "Species Concentrations and Turbulence Properties in Buoyant Methane Diffusion Flames," Journal of Heat Transfer, Vol. 106, Aug. 1985, pp. 721-727.

${ }^{20}$ Loth, E., and Faeth, G. M., "Structure of Underexpanded Round Air Jets Submerged in Water," International Journal of Multiphase Flow, Vol. 15, 1989, pp. 589-603.

${ }^{21}$ Solomon, A. S. P., Shuen, J.-S., Zhang, Q.-F., and Faeth, G. M., "Structure of Nonevaporating Sprays: I. Near-Injector Conditions and Mean Properties," AIAA Journal, Vol. 23, No. 10, 1985, pp. $1548-1555$.

${ }^{22}$ Solomon, A. S. P., Shuen, J.-S., Zhang, Q.-F., and Faeth, G. M., "Structure of Nonevaporating Sprays: II. Drop and Turbulence Properties," AIAA Journal, Vol. 23, No. 11, 1985, pp. 1724-1730.

${ }^{23}$ Simmons, H. C., "The Correlation of Drop-Size Distributions in Fuel Nozzle Sprays," Journal of Engineering for Power, Vol. 99, July 1977 , pp. 309-319.

${ }^{24}$ Levich, V. G., Physiocochemical Hydrodynamics, Prentice-Hall, Englewood Cliffs, NJ, 1962, pp. 639-646.

${ }^{25}$ Taylor, G. C., "Generation of Ripples by Wind Blowing Over a Viscous Liquid," Collected Works of G. I. Taylor 3, 1940, pp. 244254.

${ }^{26}$ Lee, D. W. , and Spencer, R. C. , "Preliminary Photomicrographic Studies of Fuel Sprays," NACA Tech Note 424, 1932; ibid, "Photomicrographic Studies of Fuel Sprays," NACA Tech. Note 454, 1933.

${ }^{27}$ Reitz, R. D., "Atomization and Other Breakup Regimes of a Liquid Jet," Ph.D. Dissertation 1375-T, Princeton Univ., 1978.

${ }^{28}$ Reitz, R. D., and Bracco, F. V., "Mechanism of Atomization of 
a Liquid Jet," Physics of Fluids, Vol. 25, Oct. 1982, pp. 1730-1742. ${ }^{29}$ Laufer, J., "The Structure of Turbulence in Fully-Developed Pipe Flow," NACA Rept. 1174, 1954.

${ }^{30}$ Clift, R., Grace, J. R., and Weber, M. E., Bubbles, Drops and Particles, Academic Press, New York, 1978, p. 346.

${ }^{31}$ Pruppacker, H. R., and Klett, J. D., Microphysics of Clouds and
Precipitation, D. Reidel Publishing Co., Boston, 1978, pp. 123-456. ${ }^{32}$ Borisov, A. A., Gel'fand, B. E., Natanzon, M. S., and Kossov, O. M., "Droplet Breakup Regimes and Criteria for Their Existence," Inzhenerno-Fizicheskii Zhurnal, Vol. 40, Jan. 1981, pp. 64-70.

${ }^{33}$ Ranger, A. A., and Nicholls, J. A., "Aerodynamic Shattering of Liquid Drops," AlAA Journal, Vol. 7, No. 2, 1969, pp. 285-290.

\title{
Commercial Opportunities in Space
}

\author{
F. Shahrokhi, C. C. Chao, and K. E. Harwell, editors
}

The applications of space research touch every facet of life-and the benefits from the commercial use of space dazzle the imagination! Commercial Opportunities in Space concentrates on present-day research and scientific developments in "generic" materials processing, effective commercialization of remote sensing, real-time satellite mapping, macromolecular crystallography, space processing of engineering materials, crystal growth techniques, molecular beam epitaxy developments, and space robotics. Experts from universities, government agencies, and industries worldwide have contributed papers on the technology available and the potential for international cooperation in the commercialization of space.

TO ORDER: Write, Phone, or FAX: American Institute of Aeronautics and Astronautics c/o Publications Customer Service, 9 Jay Gould Ct. P.O. Box 753 , Waldorf, MD 20604 Phone: $301 / 645-5643$ or 1-800/682-AIAA Dept. $415 \backsim$ FAX: 301/843-0159

Sales Tax: CA residents, $8.25 \%$; DC, 6\%. For shipping and handling add 1988

540 pp., illus. Hardback $\$ 4.75$ for $1-4$ books (call for rates for higher quanties). Orders under $\$ 50.00$ must be prepaid. Foreign orders must be prepaid. Please allow

4 weeks for delivery. Prices are subject to change without notice. AIAA Members $\$ 54.95$ Returns will be accepted within 15 days. 